\title{
De-noising SPECT/PET Images Using Cross-Scale Regularization
}

\author{
Yinpeng Jin ${ }^{1}$, Elsa D. Angelini ${ }^{1}$, Peter D. Esser ${ }^{2}$, and Andrew F. Laine ${ }^{1,2}$ \\ ${ }^{1}$ Department of Biomedical Engineering, Columbia University, New York, NY 10027 \\ ${ }^{2}$ Department of Radiology, Columbia University, New York, NY 10032 \\ $\{y j 76$, ea179, pde2, laine\}@columbia.edu
}

\begin{abstract}
De-noising of SPECT and PET images is a challenging task due to the inherent low signal-to-noise ratio of acquired data. Wavelet based multiscale denoising methods typically apply thresholding operators on sub-band coefficients to eliminate noise components in spatial-frequency space prior to reconstruction. In the case of high noise levels, detailed scales of sub-band images are usually dominated by noise which cannot be easily removed using traditional thresholding schemes. To address this issue, a cross-scale regularization scheme is introduced, which takes into account cross-scale coherence of structured signals. Preliminary results show promising performance in denoising clinical SPECT and PET images for liver and brain studies. Wavelet thresholding was also compared to denoising with a brushlet expansion. The proposed regularization scheme eliminates the need for threshold parameter settings, making the denoising process less tedious and suitable for clinical practice.
\end{abstract}

\section{Introduction}

Two of the most important functional imaging modalities, single photon emission computed tomography (SPECT) and positron emission tomography (PET) have rather limited spatial resolution and low signal-to-noise ratio. Reconstruction from projection maps acquired by a scanner faces a trade-off between contrast and signalto-noise ratio [1]. Indeed reconstruction with more spatial detail is generally performed at the cost of introducing additional noise. Current commercial systems use filtered backprojection to reconstruct that can amplify high-frequency noise components and degrade further analysis and clinical interpretation of the data. In this context, sophisticated denoising can play an important role as a pre-processing step for the benefit of clinical interpretation and possibly reduce patient dose of the radionuclide.

The majority of clinical reading platforms apply simple low-pass filtering and averaging operators as pre-processing to eliminate noise components and improve the visual quality before diagnostic interpretation of the data. This paper introduces a novel multi-scale denoising method for clinical PET and SPECT data. Denoising is performed in 3D space to take advantage of better separation of noise and signal in higher dimensions and the availability of volumetric features available in true 3D datasets. 


\section{Method}

Wavelet based multi-scale methods have been extensively used for image enhancement and denoising problems [2-6]. Unlike traditional single scale methods of analysis, which can be ad hoc for denoising, wavelet expansions offer the possibility of separating features of interest and noise components into distinct sub-band coefficients. Many traditional single scale enhancement methods can therefore be applied to each analysis sub-band image according to the signal-to-noise properties within a spatial-frequency representation.

\subsection{Dyadic Expansions and Derivative Based Spline Wavelets}

In general, the wavelet transform of a signal $f(x)$ at scale $s$ with translation $u$ is defined by [7]

$$
W f(u, s)=f * \psi_{u, s}=\int f(x) \frac{1}{\sqrt{s}} \psi^{*}\left(\frac{x-u}{s}\right) d x .
$$

A discrete wavelet transform is obtained from a continuous representation by discretizing dilation and translation parameters such that the resulting set of wavelets constitutes a frame. The dilation parameter is typically discretized by an exponential sampling with a fixed dilation step and the translation parameter by integer multiples of a dilation dependent step. Unfortunately, the resulting transform is variant under translation, a property which makes it less attractive for the analysis of non-stationary signals.

Sampling the translation parameter with the same sampling period as the input function to the transform results in a translation-invariant, but slightly redundant representation. The dyadic wavelet transform proposed by Mallat and Zhong [8] is one such representation. The dyadic wavelet transform of a function $s(x) \in L^{2}(R)$ is defined as a sequence of functions $\left\{W_{m} s(x)\right\}_{m \in Z}$, where

$$
W_{m} s(x)=s * \psi_{m}(x)=\int_{-\infty}^{+\infty} s(t) \psi_{m}(x-t) d t
$$

and $\psi_{m}(x)=2^{-m} \psi\left(2^{-m} x\right)$ is a wavelet $\psi(x)$ expanded by a dilation parameter (or scale) $2^{m}$

Discrete dyadic wavelet transform can be implemented within a hierarchical filtering scheme. For an $\mathrm{N}$-dimensional discrete dyadic wavelet transform decomposition, the wavelet coefficients (sub-band expansion) consist of $N$ components for each level (scale), which represent information along each coordinate direction at a certain scale, and a DC component, which represents the "residue" information or average energy distribution.

In [9], Laine et al. proposed an extension to the family of filters described in [8]. In this design, the wavelet could be either anti-symmetric and equal to the first derivative of some smoothing function $\theta(x)$, or symmetric and equal to the second derivative of $\theta(x)$. Under this family of wavelets, it was also found that symmetric 
(second derivative like) wavelet bases were more suitable for contrast enhancement tasks [10], whereas anti-symmetric (first derivative like) wavelet bases were more suitable for edge detection and de-noising applications [11].

In this research project, we used a first derivative of the cubic spline function (Fig. $1)$ as the wavelet basis for analysis.

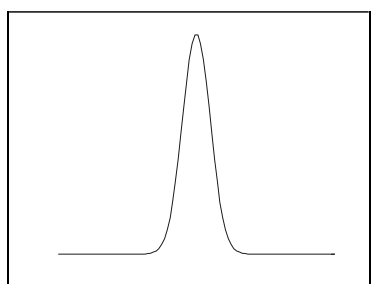

(a)

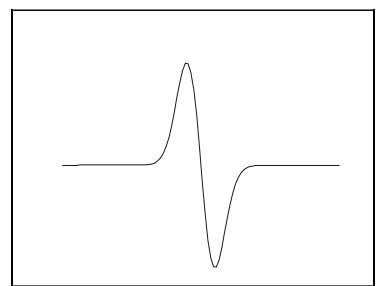

(b)

Fig. 1. (a) Cubic spline smoothing function $\theta(x)$. (b) Wavelet $\psi(x)$ of compact support defined as the derivative of the smoothing function $\theta(x)$.

\subsection{Wavelet Thresholding Operators for Denoising SPECT and PET}

In general, wavelet coefficients with larger magnitude are related to significant features such as edges in an image. Therefore, denoising can be achieved by expansion of a signal onto a set of wavelet basis functions, thresholding of the wavelet coefficients and reconstructing back to the original image (spatial) domain.

Typical threshold operators that have been used previously include hard thresholding

$$
\rho_{T}(x)= \begin{cases}x, \text { if } & |x|>T \\ 0, \text { if } & |x| \leq T\end{cases}
$$

and soft thresholding (wavelet shrinkage) [12]:

$$
\rho_{T}(x)=\left\{\begin{array}{ccc}
x-T, & \text { if } & x \geq T \\
x+T, & \text { if } & x \leq-T . \\
0, & \text { if } & |x|<T
\end{array}\right.
$$

The general framework of a filter bank implementation for over-complete multiscale denoising is schematically illustrated in Fig. 2, where a two-level decomposition is shown. The redundancy in this expansion is exploited for image denoising by first modifying transform coefficients at selected levels of spatial frequency and then reconstructing. Notice that since the DC-Cap contains most of the energy, it is usually untouched during the thresholding procedure. As shown above, the thresholding function can be implemented independent of a particular set of filters and easily incorporated into a filter bank framework to provide multi-scale denoising. Note that for N-dimensional data, each level of a wavelet expansion will have $N$ components, and the thresholding operator may be applied to each of them individually. 
Similar to the 1D case, a 3D dyadic wavelet basis can be computed from a set of three wavelets $\left(\psi^{1}, \psi^{2}, \psi^{3}\right)$ that are the partial derivatives of a smoothing function $\theta$ :

$$
\psi^{1}(x, y, z)=\frac{\partial \theta(x, y, z)}{\partial x}, \psi^{2}(x, y, z)=\frac{\partial \theta(x, y, z)}{\partial y}, \psi^{3}(x, y, z)=\frac{\partial \theta(x, y, z)}{\partial z} .
$$

We denote the dilation and translation of $\psi^{k}$ as:

$$
\psi_{j, l, m, n}^{k}(x, y, z)=\frac{1}{2^{3 j / 2}} \psi^{k}\left(\frac{x-l}{2^{j}}, \frac{y-m}{2^{j}}, \frac{z-n}{2^{j}}\right) .
$$

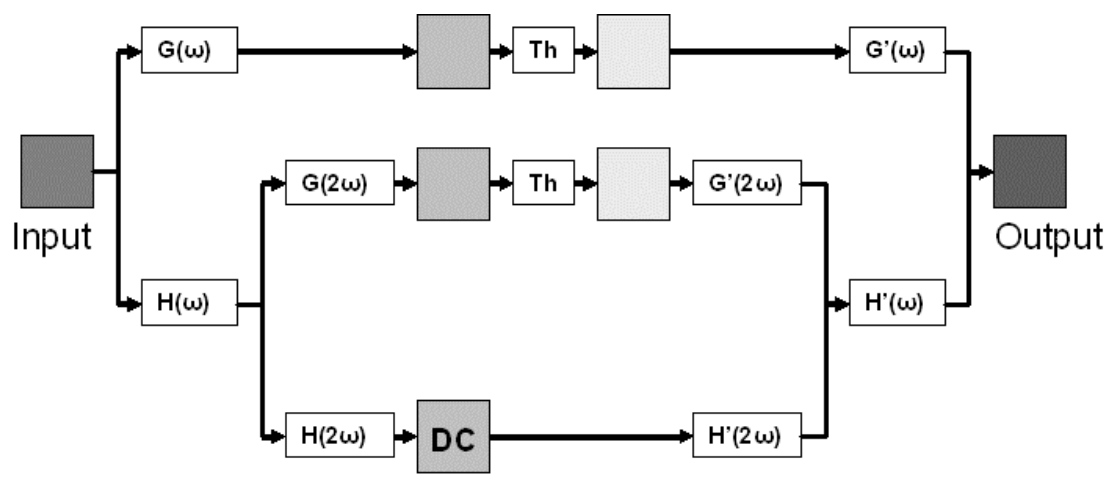

Fig. 2. Multiscale thresholding framework using a discrete dyadic wavelet transform. G, H: decomposition filters, G', H': dual-filters for reconstruction. Th: thresholding module.

Thus, the dyadic wavelet transform of a volume image $F$ at a scale $2^{j}$ has three components:

$$
T_{j}^{k} F(l, m, n)=<F, \psi_{j, l, m, n}^{k}>, \quad k=1,2,3 .
$$

Because $\left(\psi^{1}, \psi^{2}, \psi^{3}\right)$ are partial derivatives of $\theta$, the three components are proportional to the three coordinate components of the gradient vector of $F$ smoothed by a dilated version of $\theta$. From these components, one can compute the angle of the gradient vector, which indicates the direction in which the signal (a smoothed version of $F$ ) changes the most rapidly. The magnitude of this vector is proportional to the wavelet modulus:

$$
M_{j} F=\sqrt{\left|T_{j}^{1} F\right|^{2}+\left|T_{j}^{2} F\right|^{2}+\left|T_{j}^{3} F\right|^{2}} .
$$

In a previous study [13], we applied thresholding to each wavelet transform component separately. In this study, we applied a threshold operator to the magnitude of the wavelet modulus. This can be viewed as an adaptive selection of the wavelet direction in order to best correlate with the signal. A large value of wavelet modulus means that the signal changes rapidly in the neighborhood of this location, which suggests the existence of important surface boundary features. In this case, the threshold operator keeps the signal untouched. On the other hand, small values of the 
wavelet modulus mean that the signal has subtle variations in the neighborhood locations, thus indicating a more homogeneous region, possibly with a mixture of noise. In this case, the threshold operator can suppress these less significant signal variations. By treating small signal variations as noise, the adaptive threshold scheme will attenuate these components while keeping sharp signal variations that are usually correlated with important clinical structures, such as tumor boundaries.

\subsection{Cross-Scale Regularization}

Selection of threshold values for individual wavelet coefficients (or modulus, in our case) determines the efficiency of the denoising process. The threshold value should enable the process to eliminate noise components in each sub-band while avoiding over-thresholding that would destroy edge information and introduce undesirable smoothing and signal distortion.

Each analysis sub-band of wavelet expansion contains distinct spatial frequency components of the original data, and appears to contain distinct signal/noise mixtures. This suggests that different threshold values at each sub-band may be needed for optimal denoising. Even without knowing precisely the noise characteristics in a signal, it is sometimes helpful to visualize the individual sub-band images before selecting a threshold value.

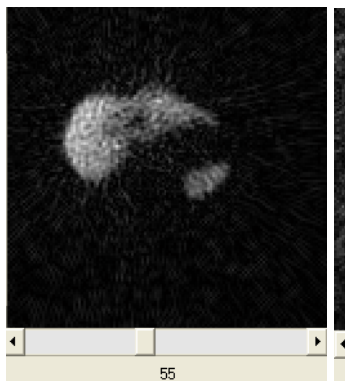

(a)

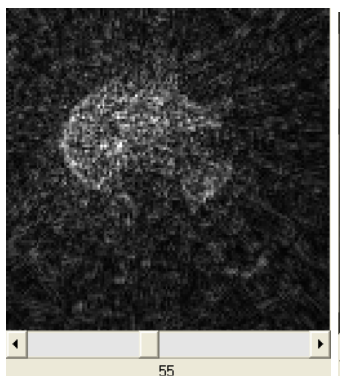

(b)

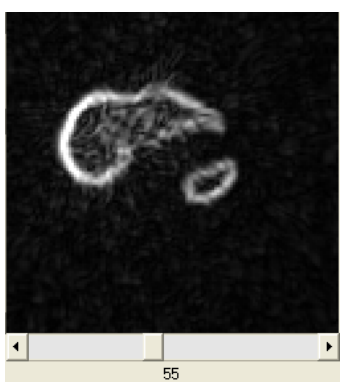

(c)

Fig. 3. (a) One slice from a reconstructed SPECT liver dataset. 3D wavelet coefficient modulus at (b) level 1 and (c) level 2.

Fig. 3 shows a display of a single slice of wavelet modulus coefficients from a 3D SPECT dataset. A two level dyadic decomposition using the first derivative of a cubic spline wavelet was applied. The first sub-band (Fig. 3 (b)) shows significantly higher noise level when compared to the second level sub-band (Fig. 3 (c)).

In dyadic wavelet decompositions, lower level sub-bands contain high-frequency components thus noise components are mostly distributed within these levels. From Fig. 3 (b), we can see that the first sub-band was dominated by noise, and that important signal information was buried within the high level noise. Without additional information or a priori knowledge of the signal, it is difficult to extract useful information via a global point-wise threshold operator as illustrated in Figure 4. On the 
other hand, the second level coefficients can be easily processed as signal components that occupy most of the higher dynamic range.

When thresholding the wavelet coefficient modulus displayed in Fig. 3 (b) to extract meaningful signal information from the noisy data, a straightforward strategy would make use of prior knowledge about the location of anatomical features of interest. Identification of these features cannot be done from simple visual inspection since the noise level is comparable or sometimes higher than wavelet coefficients modulus. In this case, shape or feature based priors can be used.

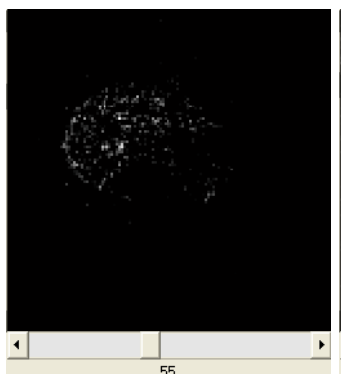

(a)

(b)

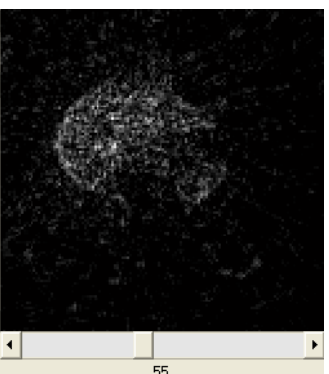

(c)

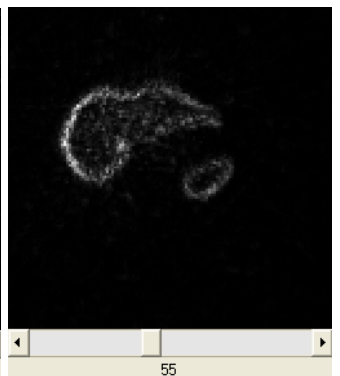

Fig. 4. Soft thresholding on level 1 wavelet modulus at level $\mathrm{T}$ : (a) $\mathrm{T}=0.1$, (b) $\mathrm{T}=0.2$. (c) Thresholded wavelet modulus after cross-scale regularization.

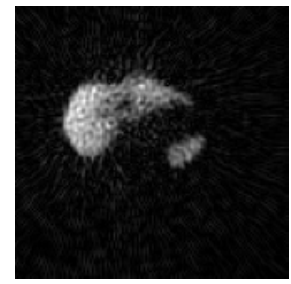

(a)

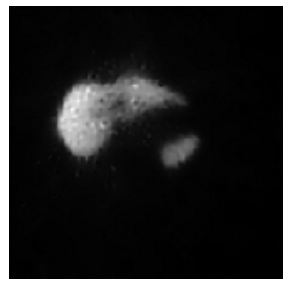

(b)

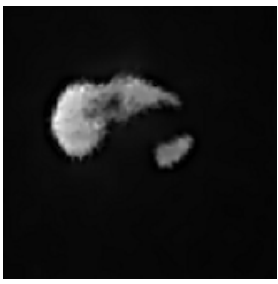

(c)

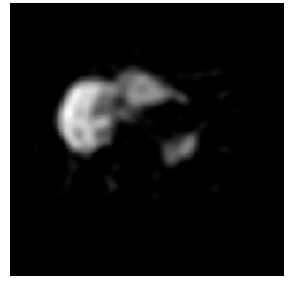

(d)

Fig. 5. (a) Slice from a liver SPECT dataset. (b-d) Slices denoised with: (b) simple softthresholding of wavelet modulus, (c) soft-thresholding with cross-scale regularization, (c) brushlet thresholding.

By using the first derivative of a smoothing function as the wavelet basis, we know that the wavelet coefficient modulus represents the gradient of the data a certain wavelet scale. Since gradient values indicate how rapidly pixel intensities change locally, it is equivalent to an edge map, where higher values represent more significant edges. Therefore, without seeking external prior information about the edges, we can easily identify anatomical features from the higher level sub-bands. This is illustrated in Fig. 3 (c) where level 2 sub-band coefficient modulus provide a good edge map estimation. By first normalizing this image to the range of $[0,1]$, we can therefore construct a prior used to regularize the first level wavelet modulus coefficients.

A simple regularization technique is to apply a voxel-wise multiplication of the prior edge map to the first level coefficients modulus. This is also equivalent to identifying coherent features across wavelet scales for determining the location of a signal. This regularization scheme was first proposed by Mallat and Zhong [14] for edge 
detection using dyadic wavelets. Fig. 5 (c) shows results using this regularization scheme for denoising of a clinical liver SPECT dataset. For comparison, denoising result using a traditional soft thresholding approach is shown in Fig. 5 (b). The proposed regularization scheme is particularly suited for images with high levels of noise. Comparing Fig. 5 (b) and (c), we see a significant improvement in image quality without losing edge information.

There are two design parameters involved in the proposed cross-scale regularization scheme. The depth of the wavelet decomposition is usually determined by the size of the sampled input data. PET and SPECT datasets typically have a $128 \times 128$ pixel matrix size, thus a three level expansion is sufficient. Regarding the selection of the levels to regularize, regularization on the first level alone is usually sufficient, since only this level is dominated by noise.

\section{Results and Discussion}

We tested the proposed denoising scheme on a 3D PET brain data set acquired with a Siemens HR+ scanner. The results are illustrated in Fig. 6 below. The current PET clinical system uses a filtered backprojection algorithm that combines a ramp filter for backprojection and a low-pass filter to suppress high-frequency noise.

Fig. 6 (b) shows a slice reconstructed on the clinical system using a Hann window with a cutoff frequency of 0.4 . To further increase signal-to-noise ratio, clinical PET systems usually average four time frames acquisitions. In addition adjacent pairs of slices are also averaged prior to display to improve image quality. While some lowpass filtering is required to remove the high levels of noise, spatial and temporal averaging typically destroys finer anatomical structures as well as edge definition.

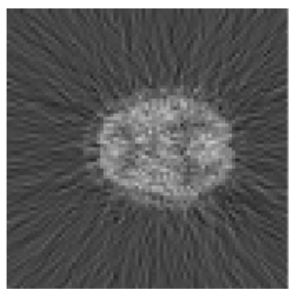

(a)

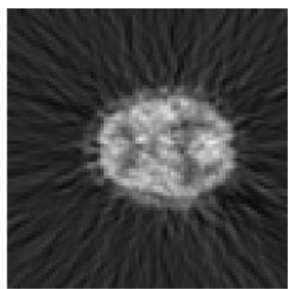

(b)

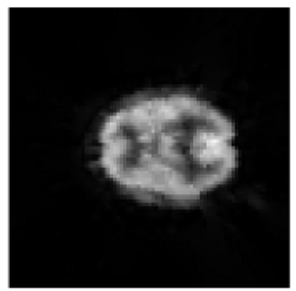

(c)

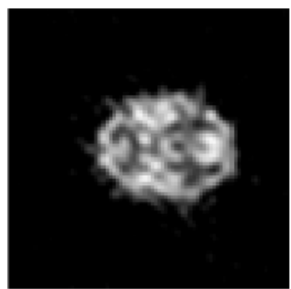

(d)

Fig. 6. Clinical PET brain data set reconstructed with: (a) a ramp filter, (b) a Hann window low pass filter (clinical settings). (c-d) Denoising of the reconstructed data in (a) with (c) wavelet thresholding with cross-scale regularization, (d) brushlet thresholding.

With SPECT and PET data, the directional texture of the noise components causes most of the standard 2D denoising methods fail since directional patterns appear as significant spatial features in 2D slices. This observation motivated the implementation of dyadic wavelet denoising in 3D space, to isolate strong 3D boundary features such as surfaces that usually correlate with true anatomical features. 
Fig. 6 (c) shows denoising results from a 3D dyadic wavelet expansion of the reconstructed data shown in

Fig. 6 (a) and thresholding with cross-scale regularization. We can visually assess that the denoising quality was significantly improved when compared to the clinical reconstruction method shown in

Fig. 6 (b), as it provided better edge definition and improved the visibility of structure.

A parallel approach investigated by our group in a previous study on SPECT brain data used a brushlet multi-scale expansion and spatially adaptive hard thresholding to remove texture noise components in 2D slices [15]. We applied this denoising algorithm to the two clinical data sets and provide sample results in Fig. 5 and

Fig. 6. We observed in these experiments very good performance of brushlet denoising at removing background noise and enhancing physiological data (with higher details of brightness levels within different structures) than with wavelet denoising but observed less spatial delineation of the anatomical contours.

\section{Future Improvement}

The cross-scale regularization method proposed in this paper may be further improved by combining other multi-scale thresholding schemes. Before regularization, second level wavelet coefficients can be "pre-processed" by independent threshold operators to improve the quality of prior information. Also, since the edge prior information in the second level wavelet coefficient modulus is not a perfect edge map, it might also attenuate weak edges. Applying a linear/nonlinear enhancement operator on the regularized term will be tested to correct for this deficiency. Finally the comparison with the texture denoising method using brushlet expansions suggests a powerful reason for fusing the two denoising methods: spline-wavelet 3D denoising for reconstruction of anatomical features (based on edge information) and brushlet 2D denoising for reconstruction of physiological information (based on texture information).

\section{References}

[1] J. M. Ollinger and J. A. Fessler, "Positron-Emission Tomography," IEEE Signal Processing Magazine, pp. 43-55, 1997.

[2] J. B. Weaver, X. Yansun, D. M. Healy, and L. D. Cromwell, "Filtering noise from images with wavelet transforms," Magnetic Resonance in Medicine, vol. 21, pp. 288-295, 1991.

[3] R. A. Devore and B. J. Lucier, "Fast Wavelet techniques for near-optimal image processing," presented at IEEE Military Communication Conference, New York, 1992.

[4] D. L. Donoho, "De-noising by soft-thresholding," IEEE Transactions on Information Theory, vol. 41, pp. 613-627, 1995.

[5] J. Fan and A. F. Laine, "Multiscale contrast enhancement and denoising in digital radiographs," in Wavelets in Medicine and Biology, A. Aldroubi and M. Unser, Eds. Boca Raton, FL: CRC Press, 1996, pp. 163-189. 
[6] A. Aldroubi and M. Unser, Wavelets in Medicine and Biology. Boca Raton, FL: CRC, 1996.

[7] S. Mallat, A Wavelet Tour of Signal Processing. San Diego, CA: Academic Press, 1998.

[8] S. Mallat and S. Zhong, "Signal characterization from multiscale edges," presented at 10th International Conference on Pattern Recognition, Atlantic City, NJ, 1990.

[9] A. F. Laine, J. Fan, and S. Schuler, "A framework for contrast enhancement by dyadic wavelet analysis," in Digital Mammography, S. M. A. A. G. Gale, D. R. Dance, and A.Y. Cairns, Ed. Amsterdam, The Netherlands: Elsevier, 1994, pp. 91-100.

[10] I. Koren and A. Laine, "A discrete dyadic wavelet transform for multidimensional feature analysis," in Time frequency and wavelets in biomedical signal processing, IEEE press series in Biomedical Engineering, M. Akay, Ed. New York: IEEE Press, 1998, pp. 425448.

[11] A. F. Laine, S. Schuler, J. Fan, and W. Huda, "Mammographic feature enhancement by multiscale analysis," IEEE Transactions on Medical Imaging, vol. 13, pp. 725-740, 1994.

[12] D. L. Donoho and I. M. Johnstone, "Threshold selection for wavelet shrinkage of noisy data," presented at 16th Annual International Conference of the IEEE Engineering in Medicine and Biology Society, 1994.

[13] J. Kalifa, A. Laine, and P. Esser, "Regularization in tomographic reconstruction using thresholding estimators," presented at SPIE Conference on Wavelet applications, San Diego, 2001.

[14] S. Mallat and S. Zhong, "Characterization of signals from multiscale edges," IEEE Transactions on Pattern Analysis and Machine Intelligence, vol. 14, pp. 710-732, 1992.

[15] E. D. Angelini, J. Kalifa, and A. F. Laine, "Harmonic multiresolution estimators for denoising and regularization of SPECT-PET data," presented at International Symposium on Biomedical Imaging, Washington, D.C., USA, 2002. 